\title{
Some Geometric Transformations on New Trees
}

\author{
M. El-Ghoul \\ Mathematics Department, Faculty of Science \\ Tanta University, Tanta, Egypt \\ Tel: 104-684-812 E-mail: m_elghoul_2009@ hotmail.com \\ SH.A.Mousa \\ Mathematics Department, Faculty of Science \\ Girl's college, Ain Shams University, Egypt \\ Tel: 166-014-989 E-mail: shereen.3010@ hotmail.com
}

\begin{abstract}
In this paper, we will introduce the tree of the new nested graph.The regular and irregular trees will be defined.

The effects of the folding and retraction on it are deduced. Some geometric transformations of the new tree into itself are discussed. The incidence and adjacent matrices of the new graph after and before these transformations are achieved.
\end{abstract}

Keywords: Graph, Tree

\section{Introduction}

A tree is a connected graph that contains no cycles. Trees sprout up as effective models in a wide variety of applications. Every connected graph $G$ admits a spanning tree, which is a tree that contains every vertex of $G$ and whose edges are edges of $G$. Every connected graph even admits a normal spanning tree.

In graph theory, tree is a connected cyclic graph. A cyclic graph which is not necessarily connected is sometimes called a forest (because it consists of trees).

We mention brief examples:

(1) Trees are useful for modeling the possible outcomes of an experiment. For example, consider an experiment in which a coin is flipped and a 6-sides die is rolled. The leaves in the tree correspond to the outcomes in the probability space for this experiment.

(2) Programmers often use tree structures to facilitate searches and sorts and to model the logic of algorithms. For instance, the logic for a program that finds the maximum of four numbers $(w, x, y, z)$ can be represented by a tree.

(3) Chemists can use trees to represent, among other things, saturated hydro-carbons chemical compounds of the form $\mathrm{C}_{n} \mathrm{H}_{2 n+2}$ (propane, for example). The bonds between the carbons and hydrogen atoms are depicted in a tree.

\section{Definitions and Background:}

(1) Let $M$ and $N$ be two Riemannian manifolds (not necessarily of the same dimension), a map $F: M \longrightarrow N$ is said to be an "Isometric folding" of $M$ into $N$ if, for each piecewise geodesic path $\gamma: I \longrightarrow M(I=[0,1] \subset R)$, the induced path $F \circ \gamma: I \longrightarrow N$ is piecewise geodesic and of the same length as $\gamma$. If $F$ not preserves lengths, then $F$ is a topological folding (A.T.White, 1973).

(2) A subset $A$ of a topological space $X$ is called a "Retract" if there exist a continuous map $R: X \longrightarrow A$ (called retraction) such that $R(a)=a, \forall a \in A$ (M.El-Ghoul, 2006).

(3) Two or more edges joining the same pair of vertices are called " multiple edges" and an edge joining a vertex to itself is called a "loop"(William S.Massey, 1967).

(4) A "connect" graph is a graph that in one piece, whereas one which splits into several pieces is " disconnected" (William S.Massey, 1967). 


\section{The main result}

We can define new types of trees:

\subsection{New tree with loop}

It is a connected graph that contains number of loops, see Fig.(1)

$<$ Figure $1>$

Where,

$A(G)=\left[\begin{array}{lllll}0 & 1 & 0 & 0 & 0 \\ 1 & 1 & 1 & 1 & 1 \\ 0 & 1 & 1 & 0 & 0 \\ 0 & 1 & 0 & 1 & 0 \\ 0 & 1 & 0 & 0 & 1\end{array}\right]$

and

$I(G)=\left[\begin{array}{llll}1 & 0 & 0 & 0 \\ 1 & 1 & 1 & 1 \\ 0 & 1 & 0 & 0 \\ 0 & 0 & 1 & 0 \\ 0 & 0 & 0 & 0\end{array}\right]$

We can make some geometric transformations on its loops:

(1) Folding:

We can make folding on loops which gives us the original tree

$<$ Figure $2>$

Such that

$A(G)=\left[\begin{array}{lllll}0 & 1 & 0 & 0 & 0 \\ 1 & 1 & 1 & 1 & 1 \\ 0 & 1 & 1 & 0 & 0 \\ 0 & 1 & 0 & 1 & 0 \\ 0 & 1 & 0 & 0 & 1\end{array}\right] \stackrel{F_{1}}{\rightarrow}\left[\begin{array}{llllllll}0 & 1 & 0 & 0 & 0 & 0 & 0 & 0 \\ 1 & 0 & 1 & 1 & 1 & 0 & 0 & 0 \\ 0 & 1 & 0 & 0 & 0 & 0 & 0 & 1 \\ 0 & 1 & 0 & 0 & 0 & 0 & 1 & 0 \\ 0 & 1 & 0 & 0 & 0 & 1 & 0 & 0 \\ 0 & 0 & 0 & 0 & 1 & 0 & 0 & 0 \\ 0 & 0 & 0 & 1 & 0 & 0 & 0 & 0 \\ 0 & 0 & 1 & 0 & 0 & 0 & 0 & 0\end{array}\right]$

and

$I(G)=\left[\begin{array}{llll}1 & 0 & 0 & 0 \\ 1 & 1 & 1 & 1 \\ 0 & 1 & 0 & 0 \\ 0 & 0 & 1 & 0 \\ 0 & 0 & 0 & 0\end{array}\right] \stackrel{F_{1}}{\rightarrow}\left[\begin{array}{ccccccc}1 & 0 & 0 & 0 & 0 & 0 & 0 \\ 1 & 1 & 1 & 1 & 0 & 0 & 0 \\ 0 & 1 & 0 & 0 & 0 & 0 & 1 \\ 0 & 0 & 1 & 0 & 0 & 1 & 0 \\ 0 & 0 & 0 & 1 & 1 & 0 & 0 \\ 0 & 0 & 0 & 1 & 0 & 0 & 0 \\ 0 & 0 & 1 & 0 & 0 & 0 & 0 \\ 0 & 1 & 0 & 0 & 0 & 0 & 0\end{array}\right]$

From the above folding $F_{1}$, we find that $F_{1}$ reduce the number of loops (vanishes) and increase the number of vertices $\left(v_{5}, v_{6}, v_{7}\right)$ and the number of edges $\left(v_{2} v_{7}, v_{3} v_{6}, v_{4} v_{5}\right)$.

(2) Retraction:

By retraction, the terminal loops vanish.

Let $R_{1}:\left(T-P_{i}\right) \longrightarrow A$, where $P_{i}$ represent the loops

$<$ Figure $3>$

Where

$A(G)=\left[\begin{array}{lllll}0 & 1 & 0 & 0 & 0 \\ 1 & 1 & 1 & 1 & 1 \\ 0 & 1 & 1 & 0 & 0 \\ 0 & 1 & 0 & 1 & 0 \\ 0 & 1 & 0 & 0 & 1\end{array}\right] \stackrel{R_{1}}{\rightarrow}\left[\begin{array}{lllll}0 & 1 & 0 & 0 & 0 \\ 1 & 1 & 1 & 1 & 1 \\ 0 & 1 & 0 & 0 & 0 \\ 0 & 1 & 0 & 0 & 0 \\ 0 & 1 & 0 & 0 & 0\end{array}\right]$ 
$I(G)=\left[\begin{array}{llll}1 & 0 & 0 & 0 \\ 1 & 1 & 1 & 1 \\ 0 & 1 & 0 & 0 \\ 0 & 0 & 1 & 0 \\ 0 & 0 & 0 & 0\end{array}\right] \stackrel{R_{1}}{\rightarrow}\left[\begin{array}{llll}1 & 0 & 0 & 0 \\ 1 & 1 & 1 & 1 \\ 0 & 1 & 0 & 0 \\ 0 & 0 & 1 & 0 \\ 0 & 0 & 0 & 0\end{array}\right]$

Note that the loops are vanished and $A(G) \neq A(R(G))$ but $I(G)=I(R(G))$.

(3) Dispersion:

$<$ Figure $4>$

Where

$A(G)=\left[\begin{array}{lllll}0 & 1 & 0 & 0 & 0 \\ 1 & 1 & 1 & 1 & 1 \\ 0 & 1 & 1 & 0 & 0 \\ 0 & 1 & 0 & 1 & 0 \\ 0 & 1 & 0 & 0 & 1\end{array}\right] \stackrel{D_{1}}{\rightarrow}\left[\begin{array}{lllllllllll}0 & 1 & 0 & 0 & 0 & 0 & 0 & 0 & 0 & 0 & 0 \\ 1 & 0 & 1 & 1 & 1 & 0 & 0 & 0 & 0 & 0 & 0 \\ 0 & 1 & 0 & 0 & 0 & 1 & 1 & 0 & 0 & 0 & 0 \\ 0 & 1 & 0 & 0 & 0 & 0 & 0 & 1 & 1 & 0 & 0 \\ 0 & 1 & 0 & 0 & 0 & 0 & 0 & 0 & 0 & 1 & 1 \\ 0 & 0 & 1 & 0 & 0 & 0 & 0 & 0 & 0 & 0 & 0 \\ 0 & 0 & 1 & 0 & 0 & 0 & 0 & 0 & 0 & 0 & 0 \\ 0 & 0 & 0 & 1 & 0 & 0 & 0 & 0 & 0 & 0 & 0 \\ 0 & 0 & 0 & 1 & 0 & 0 & 0 & 0 & 0 & 0 & 0 \\ 0 & 0 & 0 & 0 & 1 & 0 & 0 & 0 & 0 & 0 & 0 \\ 0 & 0 & 0 & 0 & 1 & 0 & 0 & 0 & 0 & 0 & 0\end{array}\right]$

and

$$
I(G)=\left[\begin{array}{llll}
1 & 0 & 0 & 0 \\
1 & 1 & 1 & 1 \\
0 & 1 & 0 & 0 \\
0 & 0 & 1 & 0 \\
0 & 0 & 0 & 0
\end{array}\right] \stackrel{D_{1}}{\rightarrow}\left[\begin{array}{cccccccccc}
1 & 0 & 0 & 0 & 0 & 0 & 0 & 0 & 0 & 0 \\
1 & 1 & 1 & 1 & 0 & 0 & 0 & 0 & 0 & 0 \\
0 & 1 & 0 & 0 & 0 & 1 & 1 & 0 & 1 & 1 \\
0 & 0 & 1 & 0 & 0 & 0 & 1 & 1 & 0 & 0 \\
0 & 0 & 0 & 1 & 1 & 1 & 0 & 0 & 0 & 0 \\
0 & 0 & 0 & 0 & 0 & 0 & 0 & 0 & 0 & 1 \\
0 & 0 & 0 & 0 & 0 & 0 & 0 & 0 & 1 & 0 \\
0 & 0 & 0 & 0 & 0 & 0 & 0 & 1 & 0 & 0 \\
0 & 0 & 0 & 0 & 0 & 0 & 1 & 0 & 0 & 0 \\
0 & 0 & 0 & 0 & 0 & 1 & 0 & 0 & 0 & 0 \\
0 & 0 & 0 & 0 & 1 & 0 & 0 & 0 & 0 & 0
\end{array}\right]
$$

The dispersion increases the number of edges and vertices.

\subsection{Pure looped tree}

Consider a tree every edge in it is a loop, see Fig.(5)

$<$ Figure $5>$

$A(G)=\left[\begin{array}{lllll}0 & 2 & 0 & 0 & 0 \\ 2 & 0 & 2 & 2 & 2 \\ 0 & 2 & 1 & 0 & 0 \\ 0 & 2 & 0 & 1 & 0 \\ 0 & 2 & 0 & 0 & 1\end{array}\right] \stackrel{F_{1}}{\rightarrow}\left[\begin{array}{llllllll}0 & 1 & 0 & 0 & 0 & 0 & 0 & 0 \\ 1 & 0 & 1 & 1 & 1 & 0 & 0 & 0 \\ 0 & 1 & 0 & 0 & 0 & 0 & 0 & 1 \\ 0 & 1 & 0 & 0 & 0 & 0 & 1 & 0 \\ 0 & 1 & 0 & 0 & 0 & 1 & 0 & 0 \\ 0 & 0 & 0 & 0 & 1 & 0 & 0 & 0 \\ 0 & 0 & 0 & 1 & 0 & 0 & 0 & 0 \\ 0 & 0 & 1 & 0 & 0 & 0 & 0 & 0\end{array}\right]$

$I(G)=\left[\begin{array}{llll}1 & 0 & 0 & 0 \\ 1 & 1 & 1 & 1 \\ 0 & 1 & 0 & 0 \\ 0 & 0 & 1 & 0 \\ 0 & 0 & 0 & 0\end{array}\right] \stackrel{F_{1}}{\rightarrow}\left[\begin{array}{lllllll}1 & 0 & 0 & 0 & 0 & 0 & 0 \\ 1 & 1 & 1 & 1 & 0 & 0 & 0 \\ 0 & 1 & 0 & 0 & 0 & 0 & 1 \\ 0 & 0 & 1 & 0 & 0 & 1 & 0 \\ 0 & 0 & 0 & 1 & 1 & 0 & 0 \\ 0 & 0 & 0 & 1 & 0 & 0 & 0 \\ 0 & 0 & 1 & 0 & 0 & 0 & 0 \\ 0 & 1 & 0 & 0 & 0 & 0 & 0\end{array}\right]$

Where $F_{1}$ is folding 
And

$<$ Figure $6>$

Where

$A(G)=\left[\begin{array}{lllll}0 & 2 & 0 & 0 & 0 \\ 2 & 0 & 2 & 2 & 2 \\ 0 & 2 & 1 & 0 & 0 \\ 0 & 2 & 0 & 1 & 0 \\ 0 & 2 & 0 & 0 & 1\end{array}\right] \stackrel{R_{1}}{\rightarrow}\left[\begin{array}{lllll}0 & 1 & 0 & 0 & 0 \\ 1 & 0 & 1 & 1 & 1 \\ 0 & 1 & 0 & 0 & 0 \\ 0 & 1 & 0 & 0 & 0 \\ 0 & 1 & 0 & 0 & 0\end{array}\right]$

$I(G)=\left[\begin{array}{llll}2 & 0 & 0 & 0 \\ 2 & 2 & 2 & 2 \\ 0 & 2 & 0 & 0 \\ 0 & 0 & 2 & 0 \\ 0 & 0 & 0 & 0\end{array}\right] \stackrel{R_{1}}{\rightarrow}\left[\begin{array}{llll}1 & 0 & 0 & 0 \\ 1 & 1 & 1 & 1 \\ 0 & 1 & 0 & 0 \\ 0 & 0 & 1 & 0 \\ 0 & 0 & 0 & 0\end{array}\right]$

And

$<$ Figure $7>$

Such that

$A(G)=\left[\begin{array}{lllll}0 & 2 & 0 & 0 & 0 \\ 2 & 0 & 2 & 2 & 2 \\ 0 & 2 & 1 & 0 & 0 \\ 0 & 2 & 0 & 1 & 0 \\ 0 & 2 & 0 & 0 & 1\end{array}\right] \stackrel{D_{1}}{\rightarrow}\left[\begin{array}{llllllllllllll}0 & 1 & 0 & 0 & 0 & 0 & 0 & 0 & 0 & 0 & 0 & 0 & 0 & 0 \\ 1 & 0 & 1 & 0 & 0 & 1 & 1 & 0 & 0 & 1 & 0 & 0 & 1 & 1 \\ 0 & 1 & 0 & 1 & 1 & 0 & 0 & 0 & 0 & 0 & 0 & 0 & 0 & 0 \\ 0 & 0 & 1 & 0 & 0 & 0 & 0 & 0 & 0 & 0 & 0 & 0 & 0 & 0 \\ 0 & 0 & 1 & 0 & 0 & 0 & 0 & 0 & 0 & 0 & 0 & 0 & 0 & 0 \\ 0 & 1 & 0 & 0 & 0 & 0 & 0 & 0 & 0 & 0 & 0 & 0 & 0 & 0 \\ 0 & 1 & 0 & 0 & 0 & 0 & 0 & 0 & 0 & 0 & 0 & 0 & 0 & 0 \\ 0 & 0 & 0 & 0 & 0 & 0 & 1 & 0 & 0 & 0 & 0 & 0 & 0 & 0 \\ 0 & 0 & 0 & 0 & 0 & 0 & 1 & 0 & 0 & 0 & 0 & 0 & 0 & 0 \\ 0 & 1 & 0 & 0 & 0 & 0 & 0 & 0 & 0 & 0 & 0 & 0 & 0 & 0 \\ 0 & 0 & 0 & 0 & 0 & 0 & 0 & 0 & 0 & 0 & 0 & 0 & 0 & 1 \\ 0 & 0 & 0 & 0 & 0 & 0 & 0 & 0 & 0 & 0 & 0 & 0 & 0 & 1 \\ 0 & 1 & 0 & 0 & 0 & 0 & 0 & 0 & 0 & 0 & 0 & 0 & 0 & 0 \\ 0 & 1 & 0 & 0 & 0 & 0 & 0 & 0 & 0 & 0 & 0 & 0 & 0 & 0\end{array}\right]$

And

$I(G)=\left[\begin{array}{llll}2 & 0 & 0 & 0 \\ 2 & 2 & 2 & 2 \\ 0 & 2 & 0 & 0 \\ 0 & 0 & 2 & 0 \\ 0 & 0 & 0 & 0\end{array}\right] \stackrel{D_{1}}{\rightarrow}\left[\begin{array}{ccccccccccccc}1 & 0 & 0 & 0 & 0 & 0 & 0 & 0 & 0 & 0 & 0 & 0 & 0 \\ 0 & 1 & 1 & 1 & 1 & 1 & 1 & 0 & 0 & 0 & 0 & 0 & 0 \\ 0 & 1 & 0 & 0 & 0 & 0 & 0 & 1 & 1 & 0 & 0 & 0 & 0 \\ 0 & 0 & 0 & 0 & 0 & 0 & 0 & 1 & 0 & 0 & 0 & 0 & 0 \\ 0 & 0 & 0 & 0 & 0 & 0 & 0 & 0 & 1 & 0 & 0 & 0 & 0 \\ 0 & 0 & 1 & 0 & 0 & 0 & 0 & 0 & 0 & 0 & 0 & 0 & 0 \\ 0 & 0 & 0 & 1 & 0 & 0 & 0 & 0 & 0 & 1 & 1 & 0 & 0 \\ 0 & 0 & 0 & 0 & 0 & 0 & 0 & 0 & 0 & 1 & 0 & 0 & 0 \\ 0 & 0 & 0 & 0 & 0 & 0 & 0 & 0 & 0 & 0 & 1 & 0 & 0 \\ 0 & 0 & 0 & 0 & 1 & 0 & 0 & 0 & 0 & 0 & 0 & 0 & 0 \\ 0 & 0 & 0 & 0 & 0 & 0 & 0 & 0 & 0 & 0 & 0 & 1 & 0 \\ 0 & 0 & 0 & 0 & 0 & 0 & 0 & 0 & 0 & 0 & 0 & 0 & 1 \\ 0 & 0 & 0 & 0 & 0 & 0 & 1 & 0 & 0 & 0 & 0 & 0 & 0 \\ 0 & 0 & 0 & 0 & 0 & 1 & 0 & 0 & 0 & 0 & 0 & 0 & 0\end{array}\right]$

Lemma:

Any geometric transformation on the new tree gives the original tree.

Now we will define another type of tree:

\subsection{General tree}

It is a tree which consists of vertices $V$, edges $E$, areas $A$ and volumes $L$

Where $V, E, A$ and $L$ are different in dimensions, such that the connection is direct, i.e. the one dimension joined with three dimension directly, or gradually, i.e. the one dimension joined with two dimension and the two dimension joined with three dimension. 
In the original graph, the vertices join between edges of the same dimension, but for the general tree the vertices joins between different dimensions.

\section{First: Direct case:}

\section{Example:}

Consider the following general tree in figure (5), where $v_{0}, v_{1} \ldots, v_{6}$ are of 0 -dimension, and $e_{0}, e_{1} \ldots ., e_{5}$ are of 1 -dimension, and $a_{0}, a_{1}$ are of 2-dimension, and $l_{0}, l_{1}$ and of 3-dimension.

$<$ Figure $8>$

Such that

$A(G)=\left[\begin{array}{lllllll}0 & 1 & 0 & 0 & 0 & 0 & 0 \\ 1 & 0 & 1 & 1 & 1 & 0 & 0 \\ 0 & 1 & 0 & 0 & 0 & 0 & 0 \\ 0 & 1 & 0 & 0 & 0 & 1 & 1 \\ 0 & 1 & 0 & 0 & 0 & 0 & 0 \\ 0 & 0 & 0 & 1 & 0 & 0 & 0 \\ 0 & 0 & 0 & 1 & 0 & 0 & 0\end{array}\right]$

Where the adjacent matrix of $G$ denoted by $A(G)$ is the $\mathrm{n}^{*} \mathrm{n}$ matrix in which the entry in row I and column $\mathrm{J}$ is the number of edges joining the vertices I and J.

$I(G)=\left[\begin{array}{llllll}1 & 0 & 0 & 0 & 0 & 0 \\ 1 & 1 & 1 & 0 & 1 & 0 \\ 0 & 1 & 0 & 0 & 0 & 0 \\ 0 & 0 & 0 & 1 & 1 & 1 \\ 0 & 0 & 1 & 0 & 0 & 0 \\ 0 & 0 & 0 & 1 & 0 & 0 \\ 0 & 0 & 0 & 0 & 0 & 1\end{array}\right]$

Where The incidence matrix of $G$ denoted by $I(G)$ is the $n * m$ binary matrix where $m_{i j}=1$ if $v_{i}$ is incident with $e_{j}$ and $m_{i j}=$ Ootherwise,

$M(G)=\left[\begin{array}{cc}0 & 0 \\ 0 & 0 \\ 0 & 0 \\ 0 & 0 \\ 0 & 1 \\ 0 & 0 \\ 1 & 0\end{array}\right]$

Where the area matrix of $G$ denoted by $M(G)$ is the $n * m$ binary matrix where $m_{i j}=1$ if $v_{i}$ is incident with $a_{i}$ and $m_{i j}=0$ otherwise

,and

$N(G)=\left[\begin{array}{ll}0 & 0 \\ 0 & 0 \\ 0 & 1 \\ 0 & 0 \\ 0 & 0 \\ 1 & 0 \\ 0 & 0\end{array}\right]$

Where the volume matrix of $G$ denoted by $N(G)$ is the $n * m$ binary matrix where $m_{i j}=1$ if $v_{i}$ is incident with $L_{i}$ and $m_{i j}=0$ otherwise.

$H(G)=\left[\begin{array}{cc}0 & 0 \\ 0 & 0 \\ 0 & 1 \\ 0 & 0 \\ 0 & 0 \\ 1 & 0\end{array}\right]$

Where the edges area matrix of $G$ denoted by $H(G)$ is the $n * m$ binary matrix where $m_{i j}=1$ if $e_{i}$ is incident with $a_{i}$ and 
$m_{i j}=0$ otherwise.

And

$J(G)=\left[\begin{array}{cc}0 & 0 \\ 0 & 1 \\ 0 & 0 \\ 1 & 0 \\ 0 & 0 \\ 0 & 0\end{array}\right]$

Where the edge volume matrix of $G$ denoted by $J(G)$ is the $n * m$ binary matrix where $m_{i j}=1$ if $e_{i}$ is incident with $L_{i}$ and $m_{i j}=0$ otherwise .

Now we will discuss the transformations for which we can obtain the usual tree from the new general tree by folding or retraction

\subsection{Folding of the general tree}

Let $F_{i}: T \longrightarrow T$, such that

$F_{i}\left(v_{n} v_{m}\right)=v_{n} v_{m}, n \neq m, i=1,2$.

$<$ Figure $9>$

Where

$A(G)=\left[\begin{array}{lllllll}0 & 1 & 0 & 0 & 0 & 0 & 0 \\ 1 & 0 & 1 & 1 & 1 & 0 & 0 \\ 0 & 1 & 0 & 0 & 0 & 0 & 0 \\ 0 & 1 & 0 & 0 & 0 & 1 & 1 \\ 0 & 1 & 0 & 0 & 0 & 0 & 0 \\ 0 & 0 & 0 & 1 & 0 & 0 & 0 \\ 0 & 0 & 0 & 1 & 0 & 0 & 0\end{array}\right] \stackrel{F_{1}}{\rightarrow}\left[\begin{array}{lllllll}0 & 1 & 0 & 0 & 0 & 0 & 0 \\ 1 & 0 & 1 & 1 & 1 & 0 & 0 \\ 0 & 1 & 0 & 0 & 0 & 0 & 0 \\ 0 & 1 & 0 & 0 & 0 & 1 & 1 \\ 0 & 1 & 0 & 0 & 0 & 0 & 0 \\ 0 & 0 & 0 & 1 & 0 & 0 & 0 \\ 0 & 0 & 0 & 1 & 0 & 0 & 0\end{array}\right] \stackrel{F_{2}}{\rightarrow}\left[\begin{array}{lllllll}0 & 1 & 0 & 0 & 0 & 0 & 0 \\ 1 & 0 & 1 & 1 & 1 & 0 & 0 \\ 0 & 1 & 0 & 0 & 0 & 0 & 0 \\ 0 & 1 & 0 & 0 & 0 & 1 & 1 \\ 0 & 1 & 0 & 0 & 0 & 0 & 0 \\ 0 & 0 & 0 & 1 & 0 & 0 & 0 \\ 0 & 0 & 0 & 1 & 0 & 0 & 0\end{array}\right]$

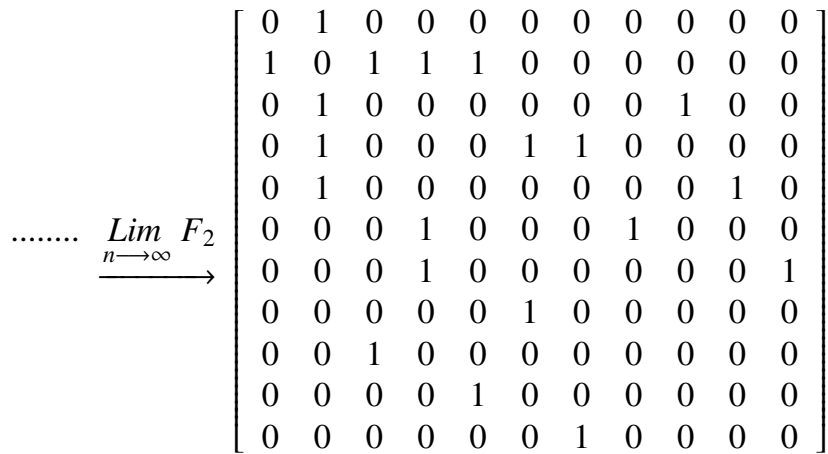

$I(G)=\left[\begin{array}{llllll}1 & 0 & 0 & 0 & 0 & 0 \\ 1 & 1 & 1 & 0 & 1 & 0 \\ 0 & 1 & 0 & 0 & 0 & 0 \\ 0 & 0 & 0 & 1 & 1 & 1 \\ 0 & 0 & 1 & 0 & 0 & 0 \\ 0 & 0 & 0 & 1 & 0 & 0 \\ 0 & 0 & 0 & 0 & 0 & 1\end{array}\right] \stackrel{F_{1}}{\rightarrow}\left[\begin{array}{llllll}1 & 0 & 0 & 0 & 0 & 0 \\ 1 & 1 & 1 & 0 & 1 & 0 \\ 0 & 1 & 0 & 0 & 0 & 0 \\ 0 & 0 & 0 & 1 & 1 & 1 \\ 0 & 0 & 1 & 0 & 0 & 0 \\ 0 & 0 & 0 & 1 & 0 & 0 \\ 0 & 0 & 0 & 0 & 0 & 1\end{array}\right] \stackrel{F_{2}}{\rightarrow}\left[\begin{array}{llllll}1 & 0 & 0 & 0 & 0 & 0 \\ 1 & 1 & 1 & 0 & 1 & 0 \\ 0 & 1 & 0 & 0 & 0 & 0 \\ 0 & 0 & 0 & 1 & 1 & 1 \\ 0 & 0 & 1 & 0 & 0 & 0 \\ 0 & 0 & 0 & 1 & 0 & 0 \\ 0 & 0 & 0 & 0 & 0 & 1\end{array}\right] \ldots \ldots \ldots$ 
$\stackrel{\operatorname{Lim}_{n \rightarrow \infty} F_{2}}{\longrightarrow}\left[\begin{array}{cccccccccc}1 & 0 & 0 & 0 & 0 & 0 & 0 & 0 & 0 & 0 \\ 1 & 1 & 1 & 0 & 1 & 0 & 0 & 0 & 0 & 0 \\ 0 & 1 & 0 & 0 & 0 & 0 & 1 & 0 & 0 & 0 \\ 0 & 0 & 0 & 1 & 1 & 1 & 0 & 0 & 0 & 0 \\ 0 & 0 & 1 & 0 & 0 & 0 & 0 & 1 & 0 & 0 \\ 0 & 0 & 0 & 1 & 0 & 0 & 0 & 0 & 1 & 0 \\ 0 & 0 & 0 & 0 & 0 & 1 & 0 & 0 & 0 & 1 \\ 0 & 0 & 0 & 0 & 0 & 0 & 0 & 0 & 1 & 0 \\ 0 & 0 & 0 & 0 & 0 & 0 & 1 & 0 & 0 & 0 \\ 0 & 0 & 0 & 0 & 0 & 0 & 0 & 1 & 0 & 0 \\ 0 & 0 & 0 & 0 & 0 & 0 & 0 & 0 & 0 & 1\end{array}\right]$

$M(G)=\left[\begin{array}{ll}0 & 0 \\ 0 & 0 \\ 0 & 0 \\ 0 & 0 \\ 0 & 1 \\ 0 & 0 \\ 1 & 0\end{array}\right] \stackrel{F_{1}}{\rightarrow}\left[\begin{array}{llll}0 & 0 & 0 & 0 \\ 0 & 0 & 0 & 0 \\ 0 & 0 & 1 & 0 \\ 0 & 0 & 0 & 1 \\ 0 & 1 & 0 & 0 \\ 0 & 0 & 0 & 0 \\ 1 & 0 & 0 & 0\end{array}\right] \stackrel{F_{2}}{\rightarrow}\left[\begin{array}{llll}0 & 0 & 0 & 0 \\ 0 & 0 & 0 & 0 \\ 0 & 0 & 1 & 0 \\ 0 & 0 & 0 & 1 \\ 0 & 1 & 0 & 0 \\ 0 & 0 & 0 & 0 \\ 1 & 0 & 0 & 0\end{array}\right]$

$N(G)=\left[\begin{array}{ll}0 & 0 \\ 0 & 0 \\ 0 & 1 \\ 0 & 0 \\ 0 & 0 \\ 1 & 0 \\ 0 & 0\end{array}\right]$

$H(G)=\left[\begin{array}{ll}0 & 0 \\ 0 & 0 \\ 0 & 1 \\ 0 & 0 \\ 0 & 0 \\ 1 & 0\end{array}\right] \stackrel{F_{1}}{\rightarrow}\left[\begin{array}{llll}0 & 0 & 0 & 0 \\ 0 & 0 & 1 & 0 \\ 0 & 1 & 0 & 0 \\ 0 & 0 & 0 & 1 \\ 0 & 0 & 0 & 0 \\ 1 & 0 & 0 & 0\end{array}\right] \stackrel{F_{2}}{\rightarrow}\left[\begin{array}{llll}0 & 0 & 0 & 0 \\ 0 & 0 & 1 & 0 \\ 0 & 1 & 0 & 0 \\ 0 & 0 & 0 & 1 \\ 0 & 0 & 0 & 0 \\ 1 & 0 & 0 & 0\end{array}\right]$

And

$J(G)=\left[\begin{array}{ll}0 & 0 \\ 0 & 1 \\ 0 & 0 \\ 1 & 0 \\ 0 & 0 \\ 0 & 0\end{array}\right]$

Note that the folding must be gradually, therefore we can deduce that the

folding of the general tree gives us the original tree.

\subsection{Retraction of the general tree}

In this section we will discuss the retraction of the new tree by making a modification on the usual retraction $R_{1}:\left(G-L_{i}\right) \longrightarrow G_{1}, R_{2}:\left(G_{1}-a_{i}\right) \longrightarrow G_{2}$

Such that,

$<$ Figure 10>

$A(G)=\left[\begin{array}{lllllll}0 & 1 & 0 & 0 & 0 & 0 & 0 \\ 1 & 0 & 1 & 1 & 1 & 0 & 0 \\ 0 & 1 & 0 & 0 & 0 & 0 & 0 \\ 0 & 1 & 0 & 0 & 0 & 1 & 1 \\ 0 & 1 & 0 & 0 & 0 & 0 & 0 \\ 0 & 0 & 0 & 1 & 0 & 0 & 0 \\ 0 & 0 & 0 & 1 & 0 & 0 & 0\end{array}\right] \stackrel{R_{1}}{\rightarrow}\left[\begin{array}{lllllll}0 & 1 & 0 & 0 & 0 & 0 & 0 \\ 1 & 0 & 1 & 1 & 1 & 0 & 0 \\ 0 & 1 & 0 & 0 & 0 & 0 & 0 \\ 0 & 1 & 0 & 0 & 0 & 1 & 1 \\ 0 & 1 & 0 & 0 & 0 & 0 & 0 \\ 0 & 0 & 0 & 1 & 0 & 0 & 0 \\ 0 & 0 & 0 & 1 & 0 & 0 & 0\end{array}\right] \stackrel{\operatorname{Lim} R_{1}}{\longrightarrow}\left[\begin{array}{ccccccc}0 & 1 & 0 & 0 & 0 & 0 & 0 \\ 1 & 0 & 1 & 1 & 1 & 0 & 0 \\ 0 & 1 & 0 & 0 & 0 & 0 & 0 \\ 0 & 1 & 0 & 0 & 0 & 1 & 1 \\ 0 & 1 & 0 & 0 & 0 & 0 & 0 \\ 0 & 0 & 0 & 1 & 0 & 0 & 0 \\ 0 & 0 & 0 & 1 & 0 & 0 & 0\end{array}\right]$ 
$\stackrel{R_{2}}{\rightarrow}\left[\begin{array}{lllllll}0 & 1 & 0 & 0 & 0 & 0 & 0 \\ 1 & 0 & 1 & 1 & 1 & 0 & 0 \\ 0 & 1 & 0 & 0 & 0 & 0 & 0 \\ 0 & 1 & 0 & 0 & 0 & 1 & 1 \\ 0 & 1 & 0 & 0 & 0 & 0 & 0 \\ 0 & 0 & 0 & 1 & 0 & 0 & 0 \\ 0 & 0 & 0 & 1 & 0 & 0 & 0\end{array}\right] \stackrel{\operatorname{Lim}}{\stackrel{\operatorname{Lim}}{\rightarrow}} R_{2}\left[\begin{array}{lllllll}0 & 1 & 0 & 0 & 0 & 0 & 0 \\ 1 & 0 & 1 & 1 & 1 & 0 & 0 \\ 0 & 1 & 0 & 0 & 0 & 0 & 0 \\ 0 & 1 & 0 & 0 & 0 & 1 & 1 \\ 0 & 1 & 0 & 0 & 0 & 0 & 0 \\ 0 & 0 & 0 & 1 & 0 & 0 & 0 \\ 0 & 0 & 0 & 1 & 0 & 0 & 0\end{array}\right]$

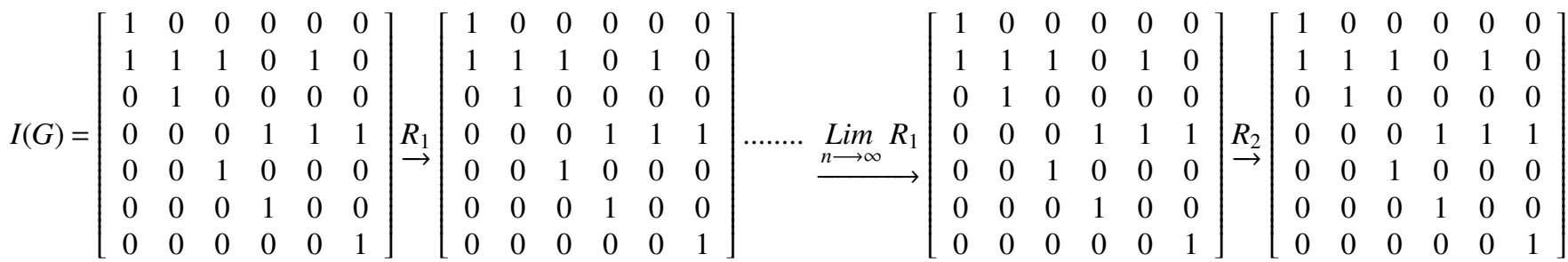

$\stackrel{\operatorname{Lim}_{n \rightarrow \infty} R_{2}}{\longrightarrow}\left[\begin{array}{llllll}1 & 0 & 0 & 0 & 0 & 0 \\ 1 & 1 & 1 & 0 & 1 & 0 \\ 0 & 1 & 0 & 0 & 0 & 0 \\ 0 & 0 & 0 & 1 & 1 & 1 \\ 0 & 0 & 1 & 0 & 0 & 0 \\ 0 & 0 & 0 & 1 & 0 & 0 \\ 0 & 0 & 0 & 0 & 0 & 1\end{array}\right]$

$M(G)=\left[\begin{array}{ll}0 & 0 \\ 0 & 0 \\ 0 & 0 \\ 0 & 0 \\ 0 & 1 \\ 0 & 0 \\ 1 & 0\end{array}\right] \stackrel{R_{1}}{\rightarrow}\left[\begin{array}{ll}0 & 0 \\ 0 & 0 \\ 0 & 0 \\ 0 & 0 \\ 0 & 1 \\ 0 & 0 \\ 1 & 0\end{array}\right] \cdots \cdots \stackrel{\operatorname{Lim}}{\longrightarrow} R_{1}\left[\begin{array}{ll}0 & 0 \\ 0 & 0 \\ 0 & 0 \\ 0 & 0 \\ 0 & 1 \\ 0 & 0 \\ 1 & 0\end{array}\right] \stackrel{R_{2}}{\rightarrow}\left[\begin{array}{ll}0 & 0 \\ 0 & 0 \\ 0 & 0 \\ 0 & 0 \\ 0 & 1 \\ 0 & 0 \\ 1 & 0\end{array}\right]$

$N(G)=\left[\begin{array}{ll}0 & 0 \\ 0 & 0 \\ 0 & 1 \\ 0 & 0 \\ 0 & 0 \\ 1 & 0 \\ 0 & 0\end{array}\right] \stackrel{R_{1}}{\rightarrow}\left[\begin{array}{ll}0 & 0 \\ 0 & 0 \\ 0 & 1 \\ 0 & 0 \\ 0 & 0 \\ 1 & 0 \\ 0 & 0\end{array}\right]$

$H(G)=\left[\begin{array}{cc}0 & 0 \\ 0 & 0 \\ 0 & 1 \\ 0 & 0 \\ 0 & 0 \\ 1 & 0\end{array}\right] \stackrel{R_{1}}{\rightarrow}\left[\begin{array}{ll}0 & 0 \\ 0 & 0 \\ 0 & 1 \\ 0 & 0 \\ 0 & 0 \\ 1 & 0\end{array}\right] \cdots \cdots \stackrel{\operatorname{Lim} R_{1}}{\longrightarrow}\left[\begin{array}{ll}0 & 0 \\ 0 & 0 \\ 0 & 1 \\ 0 & 0 \\ 0 & 0 \\ 1 & 0\end{array}\right] \stackrel{R_{2}}{\rightarrow}\left[\begin{array}{ll}0 & 0 \\ 0 & 0 \\ 0 & 1 \\ 0 & 0 \\ 0 & 0 \\ 1 & 0\end{array}\right]$

And

$J(G)=\left[\begin{array}{ll}0 & 0 \\ 0 & 1 \\ 0 & 0 \\ 1 & 0 \\ 0 & 0 \\ 0 & 0\end{array}\right] \stackrel{R_{1}}{\rightarrow}\left[\begin{array}{ll}0 & 0 \\ 0 & 1 \\ 0 & 0 \\ 1 & 0 \\ 0 & 0 \\ 0 & 0\end{array}\right]$

Second: Gradually case:

Example:

In this case the connection between edges are vertices and 1-dimension by 3-dimension through 2-dimension.

$<$ Figure $11>$ 
By folding the graph

$<$ Figure $>$

Such that

$A(G)=\left[\begin{array}{lllll}0 & 1 & 0 & 0 & 0 \\ 1 & 0 & 1 & 1 & 1 \\ 0 & 1 & 0 & 0 & 0 \\ 0 & 1 & 0 & 0 & 0 \\ 0 & 1 & 0 & 0 & 0\end{array}\right] \stackrel{F_{1}}{\rightarrow}\left[\begin{array}{lllll}0 & 1 & 0 & 0 & 0 \\ 1 & 0 & 1 & 1 & 1 \\ 0 & 1 & 0 & 0 & 0 \\ 0 & 1 & 0 & 0 & 0 \\ 0 & 1 & 0 & 0 & 0\end{array}\right] \stackrel{F_{2}}{\rightarrow}\left[\begin{array}{lllll}0 & 1 & 0 & 0 & 0 \\ 1 & 0 & 1 & 1 & 1 \\ 0 & 1 & 0 & 0 & 0 \\ 0 & 1 & 0 & 0 & 0 \\ 0 & 1 & 0 & 0 & 0\end{array}\right] \ldots \ldots \underset{n \rightarrow \infty}{\operatorname{Lim}} F_{2}\left[\begin{array}{cccccccc}0 & 1 & 0 & 0 & 0 & 0 & 0 & 0 \\ 1 & 0 & 1 & 1 & 1 & 0 & 0 & 0 \\ 0 & 1 & 0 & 0 & 0 & 0 & 0 & 0 \\ 0 & 1 & 0 & 0 & 0 & 1 & 0 & 0 \\ 0 & 1 & 0 & 0 & 0 & 0 & 1 & 0 \\ 0 & 0 & 0 & 1 & 0 & 0 & 0 & 0 \\ 0 & 0 & 0 & 0 & 1 & 0 & 0 & 0 \\ 0 & 0 & 1 & 0 & 0 & 0 & 0 & 0\end{array}\right]$

$I(G)=\left[\begin{array}{llll}1 & 0 & 0 & 0 \\ 1 & 1 & 1 & 1 \\ 0 & 1 & 0 & 0 \\ 0 & 0 & 0 & 1 \\ 0 & 0 & 1 & 0\end{array}\right] \stackrel{F_{1}}{\rightarrow}\left[\begin{array}{llll}1 & 0 & 0 & 0 \\ 1 & 1 & 1 & 1 \\ 0 & 1 & 0 & 0 \\ 0 & 0 & 0 & 1 \\ 0 & 0 & 1 & 0\end{array}\right] \stackrel{F_{2}}{\rightarrow}\left[\begin{array}{cccc}1 & 0 & 0 & 0 \\ 1 & 1 & 1 & 1 \\ 0 & 1 & 0 & 0 \\ 0 & 0 & 0 & 1 \\ 0 & 0 & 1 & 0\end{array}\right] \cdots \stackrel{\operatorname{Lim}}{\longrightarrow} F_{2}\left[\begin{array}{ccccccc}1 & 0 & 0 & 0 & 0 & 0 & 0 \\ 1 & 1 & 1 & 1 & 0 & 0 & 0 \\ 0 & 1 & 0 & 0 & 1 & 0 & 0 \\ 0 & 0 & 0 & 1 & 0 & 0 & 1 \\ 0 & 0 & 1 & 0 & 0 & 1 & 0 \\ 0 & 0 & 0 & 0 & 0 & 0 & 1 \\ 0 & 0 & 0 & 0 & 0 & 1 & 0 \\ 0 & 0 & 0 & 0 & 1 & 0 & 0\end{array}\right]$

$M(G)=\left[\begin{array}{ccc}0 & 0 & 0 \\ 0 & 0 & 0 \\ 0 & 1 & 0 \\ 1 & 0 & 0 \\ 0 & 0 & 1\end{array}\right] \stackrel{F_{1}}{\rightarrow}\left[\begin{array}{llll}0 & 0 & 0 & 0 \\ 0 & 0 & 0 & 0 \\ 0 & 0 & 1 & 0 \\ 0 & 0 & 0 & 0 \\ 0 & 0 & 1 & 1\end{array}\right] \stackrel{F_{2}}{\rightarrow}\left[\begin{array}{llll}0 & 0 & 0 & 0 \\ 0 & 0 & 0 & 0 \\ 0 & 0 & 1 & 0 \\ 0 & 0 & 0 & 0 \\ 0 & 0 & 1 & 1\end{array}\right]$

$N(G)=\left[\begin{array}{l}0 \\ 0 \\ 0 \\ 0 \\ 1\end{array}\right]$

$H(G)=\left[\begin{array}{lll}0 & 0 & 0 \\ 0 & 1 & 0 \\ 0 & 0 & 1 \\ 1 & 0 & 0\end{array}\right] \stackrel{F_{1}}{\rightarrow}\left[\begin{array}{llll}0 & 0 & 0 & 0 \\ 0 & 1 & 0 & 0 \\ 0 & 0 & 1 & 1 \\ 1 & 0 & 0 & 0\end{array}\right] \stackrel{F_{2}}{\rightarrow}\left[\begin{array}{llll}0 & 0 & 0 & 0 \\ 0 & 1 & 0 & 0 \\ 0 & 0 & 1 & 1 \\ 1 & 0 & 0 & 0\end{array}\right]$

And

$J(G)=\left[\begin{array}{l}0 \\ 0 \\ 1 \\ 0\end{array}\right]$

By retraction the graph in Fig. (11) 

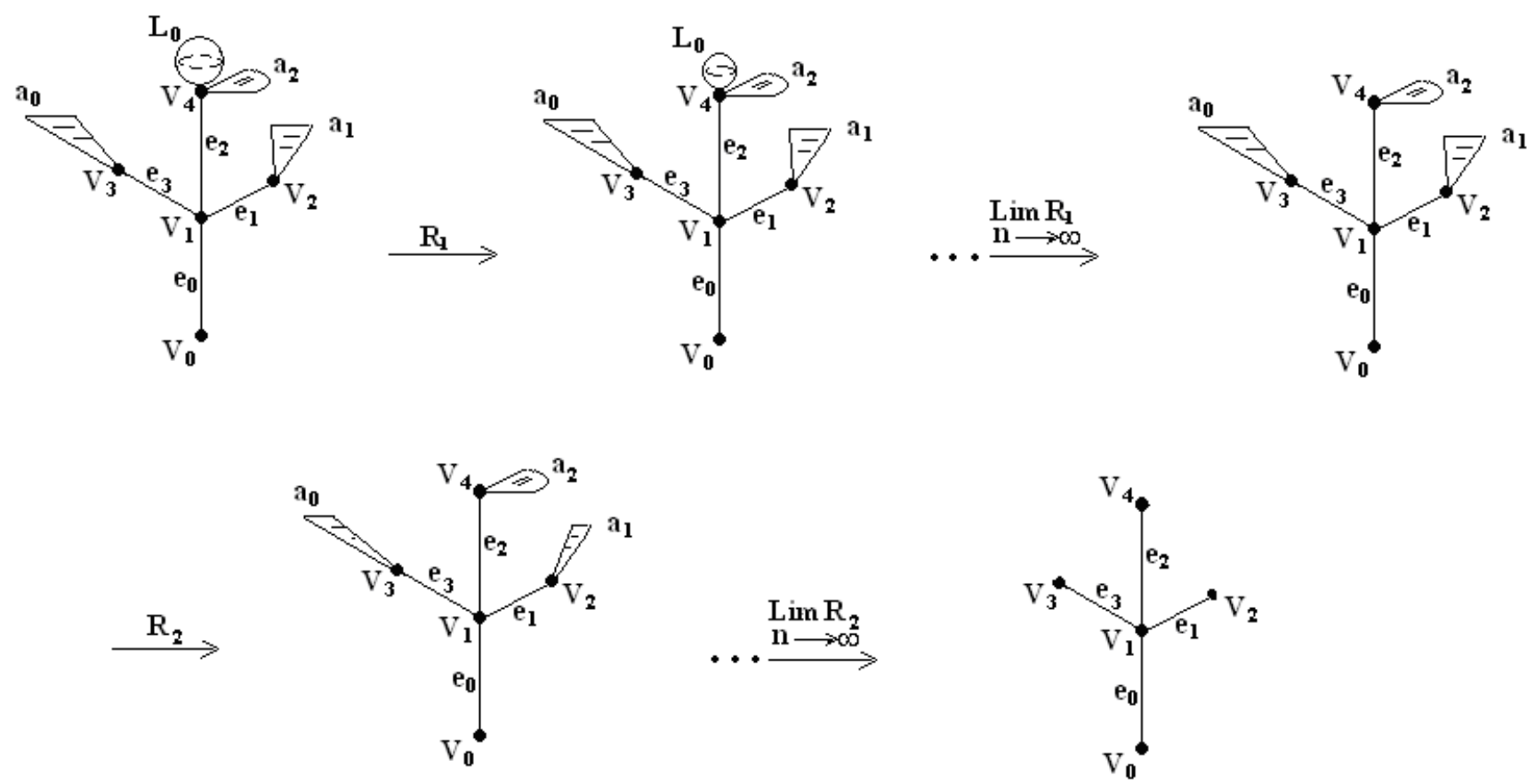

Then

$$
\begin{aligned}
& A(G)=\left[\begin{array}{lllll}
0 & 1 & 0 & 0 & 0 \\
1 & 0 & 1 & 1 & 1 \\
0 & 1 & 0 & 0 & 0 \\
0 & 1 & 0 & 0 & 0 \\
0 & 1 & 0 & 0 & 0
\end{array}\right] \stackrel{R_{1}}{\rightarrow}\left[\begin{array}{lllll}
0 & 1 & 0 & 0 & 0 \\
1 & 0 & 1 & 1 & 1 \\
0 & 1 & 0 & 0 & 0 \\
0 & 1 & 0 & 0 & 0 \\
0 & 1 & 0 & 0 & 0
\end{array}\right] \ldots \ldots \underset{n \rightarrow \infty}{\operatorname{Lim}} R_{1}\left[\begin{array}{lllll}
0 & 1 & 0 & 0 & 0 \\
1 & 0 & 1 & 1 & 1 \\
0 & 1 & 0 & 0 & 0 \\
0 & 1 & 0 & 0 & 0 \\
0 & 1 & 0 & 0 & 0
\end{array}\right] \stackrel{R_{2}}{\rightarrow}\left[\begin{array}{ccccc}
0 & 1 & 0 & 0 & 0 \\
1 & 0 & 1 & 1 & 1 \\
0 & 1 & 0 & 0 & 0 \\
0 & 1 & 0 & 0 & 0 \\
0 & 1 & 0 & 0 & 0
\end{array}\right] \\
& \stackrel{\operatorname{Lim}_{n \rightarrow \infty}}{\longrightarrow} R_{2}\left[\begin{array}{lllll}
0 & 1 & 0 & 0 & 0 \\
1 & 0 & 1 & 1 & 1 \\
0 & 1 & 0 & 0 & 0 \\
0 & 1 & 0 & 0 & 0 \\
0 & 1 & 0 & 0 & 0
\end{array}\right]
\end{aligned}
$$$$
I(G)=\left[\begin{array}{llll}
1 & 0 & 0 & 0 \\
1 & 1 & 1 & 1 \\
0 & 1 & 0 & 0 \\
0 & 0 & 0 & 1 \\
0 & 0 & 1 & 0
\end{array}\right] \stackrel{R_{1}}{\rightarrow}\left[\begin{array}{llll}
1 & 0 & 0 & 0 \\
1 & 1 & 1 & 1 \\
0 & 1 & 0 & 0 \\
0 & 0 & 0 & 1 \\
0 & 0 & 1 & 0
\end{array}\right] \cdots \cdots
$$$$
\stackrel{\operatorname{Lim}_{n \rightarrow \infty} R_{1}}{\longrightarrow}\left[\begin{array}{llll}
1 & 0 & 0 & 0 \\
1 & 1 & 1 & 1 \\
0 & 1 & 0 & 0 \\
0 & 0 & 0 & 1 \\
0 & 0 & 1 & 0
\end{array}\right] \stackrel{R_{2}}{\rightarrow}\left[\begin{array}{llll}
1 & 0 & 0 & 0 \\
1 & 1 & 1 & 1 \\
0 & 1 & 0 & 0 \\
0 & 0 & 0 & 1 \\
0 & 0 & 1 & 0
\end{array}\right]
$$$$
\stackrel{\operatorname{Lim}_{n \rightarrow \infty} R_{2}}{\longrightarrow}\left[\begin{array}{llll}
1 & 0 & 0 & 0 \\
1 & 1 & 1 & 1 \\
0 & 1 & 0 & 0 \\
0 & 0 & 0 & 1 \\
0 & 0 & 1 & 0
\end{array}\right]
$$$$
M(G)=\left[\begin{array}{lll}
0 & 0 & 0 \\
0 & 0 & 0 \\
0 & 1 & 0 \\
1 & 0 & 0 \\
0 & 0 & 1
\end{array}\right] \stackrel{R_{1}}{\rightarrow}\left[\begin{array}{lll}
0 & 0 & 0 \\
0 & 0 & 0 \\
0 & 1 & 0 \\
1 & 0 & 0 \\
0 & 0 & 1
\end{array}\right] \cdots \cdots \underset{n \rightarrow \infty}{\stackrel{\operatorname{Lim}}{\rightarrow}} R_{1}\left[\begin{array}{lll}
0 & 0 & 0 \\
0 & 0 & 0 \\
0 & 1 & 0 \\
1 & 0 & 0 \\
0 & 0 & 1
\end{array}\right] \stackrel{R_{2}}{\rightarrow}\left[\begin{array}{lll}
0 & 0 & 0 \\
0 & 0 & 0 \\
0 & 1 & 0 \\
1 & 0 & 0 \\
0 & 0 & 1
\end{array}\right]
$$ 
$N(G)=\left[\begin{array}{l}0 \\ 0 \\ 0 \\ 0 \\ 1\end{array}\right] \stackrel{R_{1}}{\rightarrow}\left[\begin{array}{l}0 \\ 0 \\ 0 \\ 0 \\ 1\end{array}\right]$

$H(G)=\left[\begin{array}{ccc}0 & 0 & 0 \\ 0 & 1 & 0 \\ 0 & 0 & 1 \\ 1 & 0 & 0\end{array}\right] \stackrel{R_{1}}{\rightarrow}\left[\begin{array}{lll}0 & 0 & 0 \\ 0 & 1 & 0 \\ 0 & 0 & 1 \\ 1 & 0 & 0\end{array}\right]$

And

$J(G)=\left[\begin{array}{l}0 \\ 0 \\ 1 \\ 0\end{array}\right] \stackrel{R_{1}}{\rightarrow}\left[\begin{array}{l}0 \\ 0 \\ 1 \\ 0\end{array}\right]$

\section{Lemma:}

The limit of folding and retraction of the general tree is the usual tree.

\section{Application:}

(1) The tree of orange can be represented as the new tree. The fruit represent volume, the leaves represent area, and the tree swing represents 1-dimension.

(2) The prickly pear tree is a kind of the new tree, as shown in the figure

$<$ Figure 13>

\section{References}

A.Gibbson. (1985). Alogrithmic graph theory. Cambridge University Press,Cambridge, UK.

A.T.White. (1973). Graphs, groups and surfaces.Amsterdam. North-Holland Publishing company.

J.L.Gross, T.W.Tucker. (1987). Topological graph theory. Jon Wiley and Sons, Inc, Canada.

M.El-Ghoul, A.El-Ahmady, H.Rafat. (2004). Folding-retraction of chaotic dynamical manifold and the VAC of vacum fluctuation. Chaos, Solitions and Fractals, UK, 20, 209-217.

M.El-Ghoul, A.El-Ahmady, T.Homda. (2006). Retraction of simplicial complexes. International Journal of Applied Mathematics and Statistics, Vol. 4(J06), 54-67, India.

P.J.Giblin. (1977). Graphs, surfaces and homology, an introduction to algebraic topology. Champman and Hall Ltd, London.

R.J.Wilson. (1972). Introduction to graph theory. Oliver and Boyed, Edinburgh.

R.J.Wilson, J.J.Watkins. (1990). Graph, an introductory approach, a fist course in discrete mathematics. Jon Wiley and Sons Inc, Canda.

William S.Massey. (1967). Algebraic Topology, an introduction. Harcourt, Brace \& world, Inc., NewYork U.S.A.

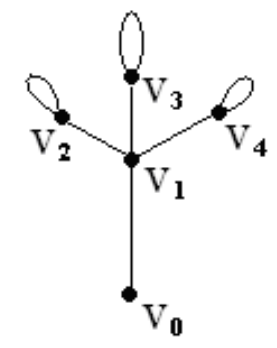

Figure 1. 

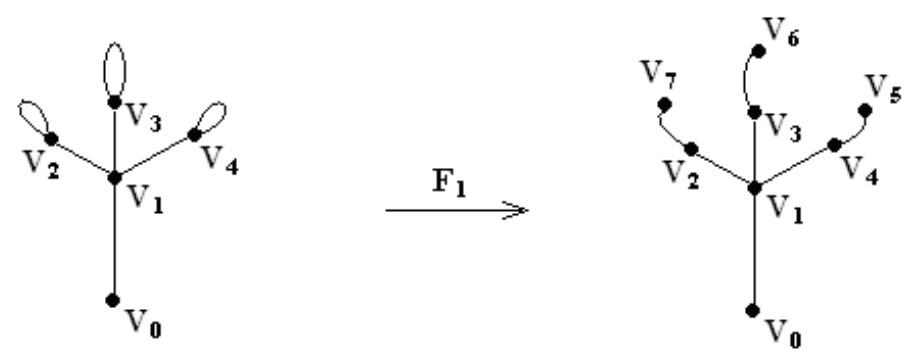

Figure 2.
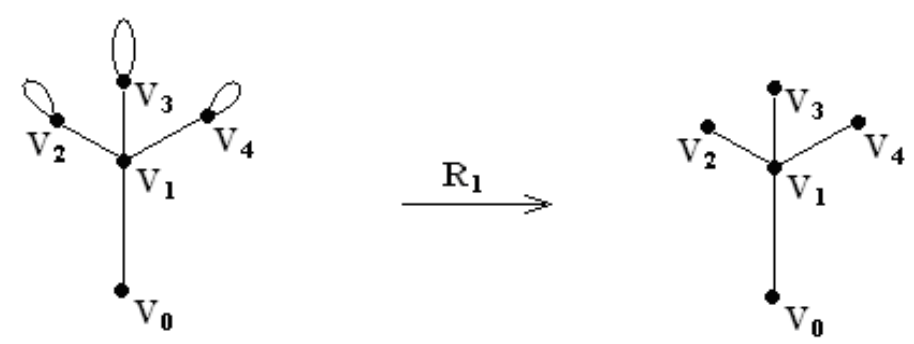

Figure 3.
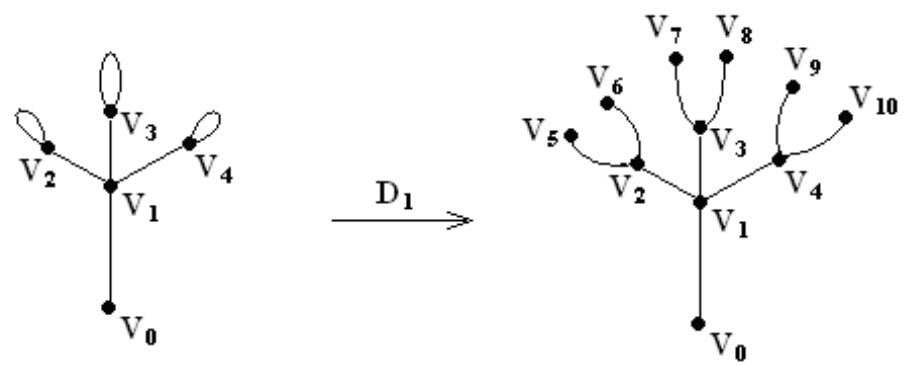

Figure 4.
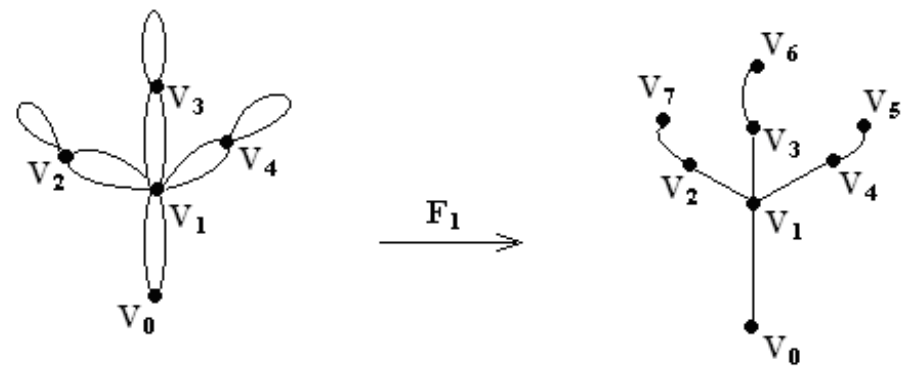

Figure 5.
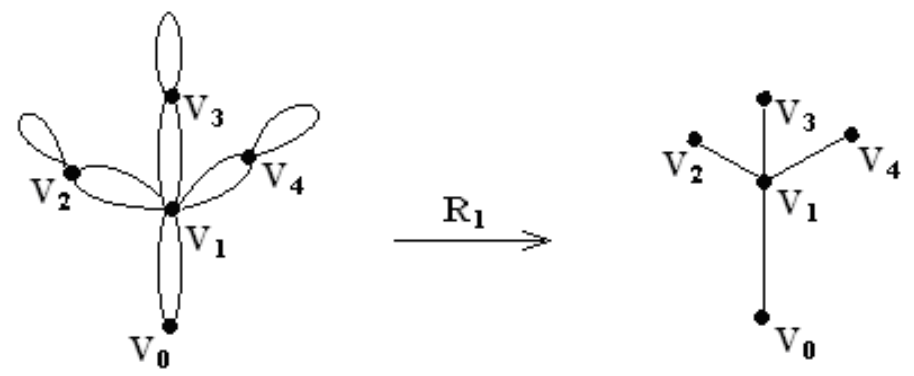

Figure 6. 

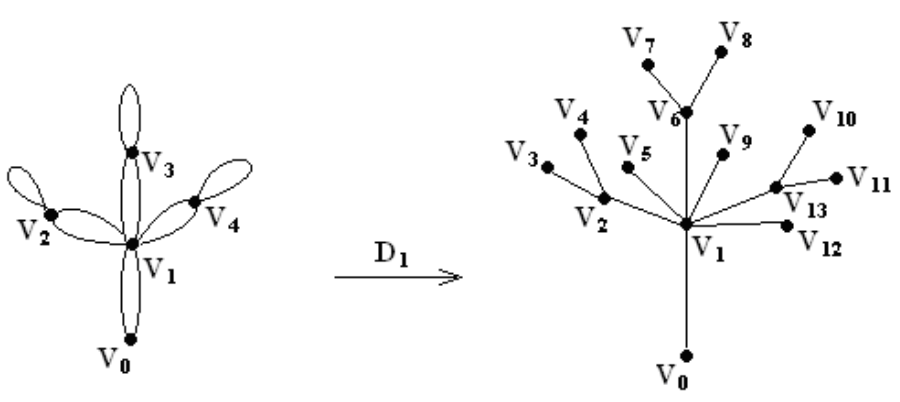

Figure 7.

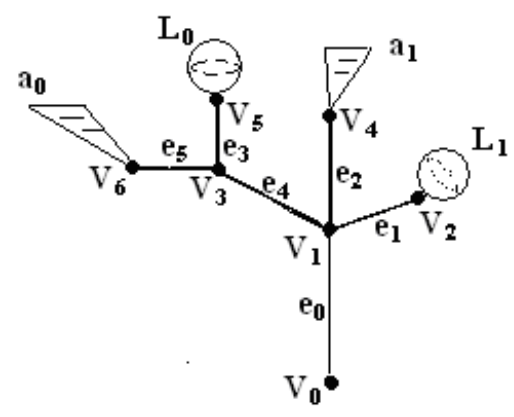

Figure 8.
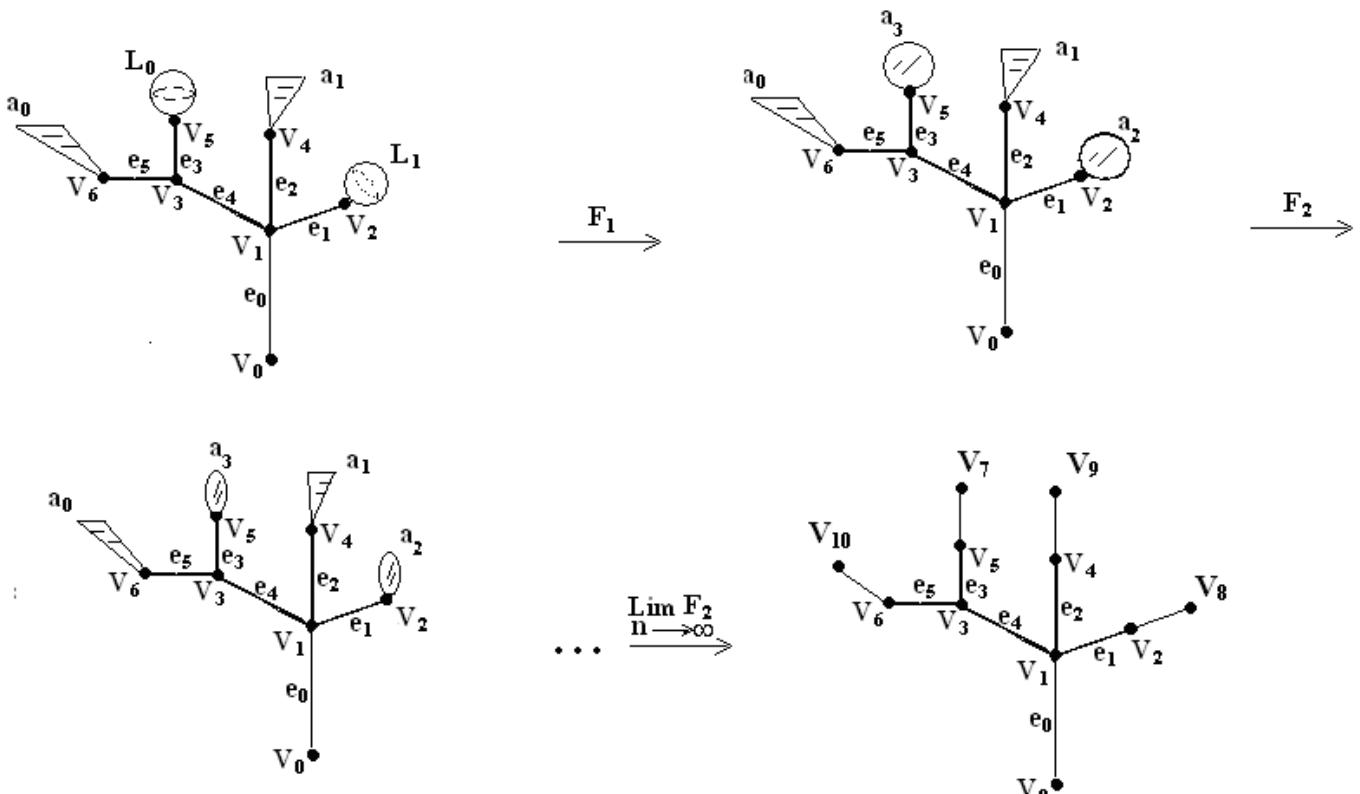

Figure 9. 

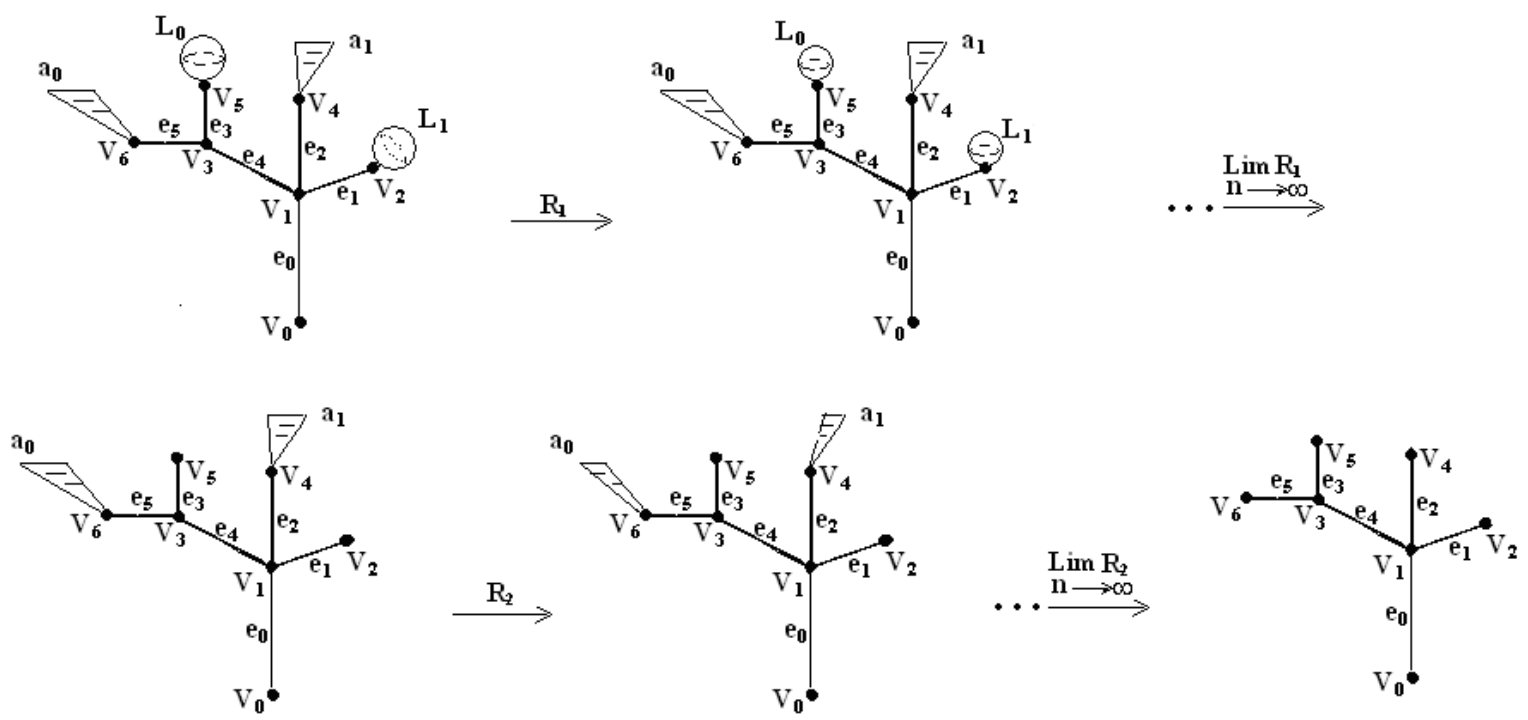

Figure 10.

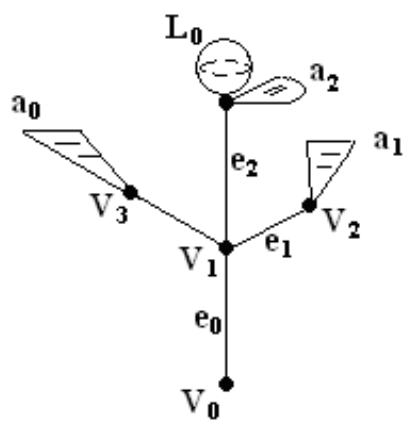

Figure 11.
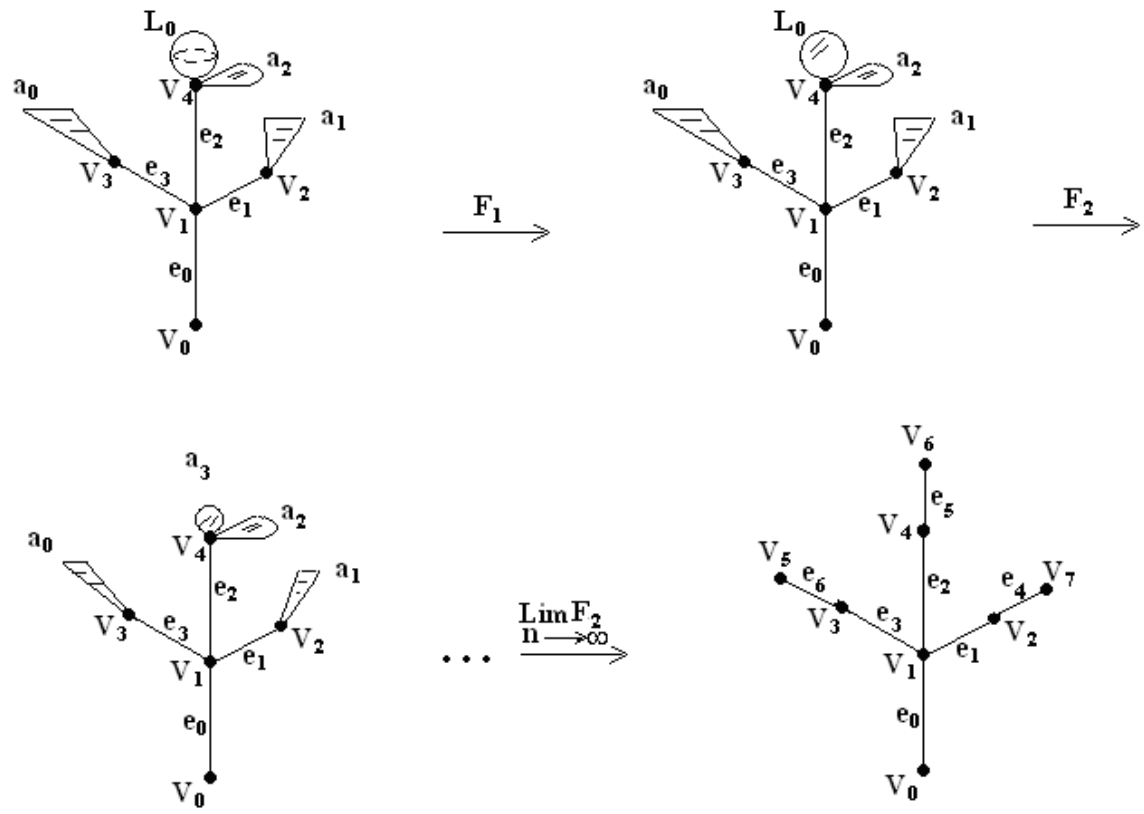

Figure 12. 


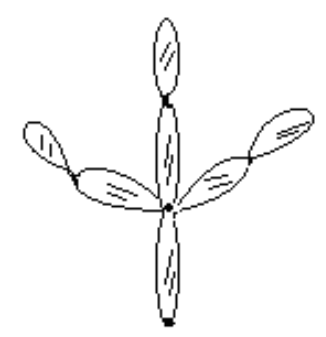

Figure 13. 